\title{
Republikanische Personennamen. Eine anthroponymische Studie zur Französischen Revolution
}

Pierre-Henri Billy

\section{Q OpenEdition Journals}

Édition électronique

URL : https://journals.openedition.org/ahrf/998

DOI : $10.4000 /$ ahrf.998

ISSN : 1952-403X

Éditeur :

Armand Colin, Société des études robespierristes

Édition imprimée

Date de publication : 1 décembre 2000

Pagination : 155-157

ISSN : 0003-4436

Référence électronique

Pierre-Henri Billy, «Republikanische Personennamen. Eine anthroponymische Studie zur Französischen Revolution », Annales historiques de la Révolution française [En ligne], 322 | octobredécembre 2000, mis en ligne le 26 avril 2006, consulté le 23 avril 2022. URL : http:// journals.openedition.org/ahrf/998; DOI : https://doi.org/10.4000/ahrf.998

Ce document a été généré automatiquement le 23 avril 2022

Tous droits réservés 


\title{
Republikanische Personennamen. Eine anthroponymische Studie zur Französischen Revolution
}

\author{
Pierre-Henri Billy
}

\section{RÉFÉRENCE}

Nicoline Hörsch, Republikanische Personennamen. Eine anthroponymische Studie zur Französischen Revolution, Tübingen, Max Niemeyer Verag (Beihefte zur Zeitschrift für romanische Philologie, 258), 1994, XVI-624 pages, $242 \mathrm{DM}^{1}$

1 Cette volumineuse thèse porte sur les prénoms révolutionnaires en France. Elle est le premier vaste essai sur le phénomène, embrassant tout le phénomène autant que faire se peut : l'on doit donc saluer le remarquable effort de l'auteur.

L'ouvrage est constitué de six parties dont :

3 1.Wege zu einer republikanischen Namenphilosophie [1-61]

$4 \quad$ 2.Untersuchung des Namenmaterials [62-343]

5 3.Datenauswertungen [344-382]

$6 \quad$ 4.Pflicht zur Namenfreiheit [383-400]

$7 \quad$ 5.Ausklang einer kulturellen Revolution [401-418]

8 Le fond même appelle quelques remarques d'ordre méthodologique,

9 Sur la terminologie d'abord. Ce qu'en France nous appelons «prénoms révolutionnaires », l'auteur les nomme « republikanische Personennamen ». Pourquoi, l'auteur ne le dit pas. "Republikanisch" a trait au seul caractère institutionnel du nouveau régime qui a succédé à la Monarchie française et très peu de prénoms font référence à ce caractère républicain. "Revolutionär ", adjectif repris à notre langue avec le même sémantisme, a trait en revanche au caractère politique et idéologique du 
nouveau régime et beaucoup de prénoms y font au rebours référence, portés notamment par cette vague sociale, cette ambiance éminemment populaire.

Sur la définition du sujet ensuite. L'auteur n'a pas pris soin de le définir réellement: certes, la chose est difficile, mais passer sous silence les phénomènes linguistiques qu'on appelle sémantisme, dénotation, connotation et motivation pour définir le " prénom révolutionnaire ", et ainsi le délimiter, nous paraît ennuyeux car c'est bien de là que découle toute analyse. Ce qui explique la suite.

11 Sur la nomenclature enfin. L'auteur n'a utilisé que la littérature dite secondaire, articles présentant parfois des listes incomplètes de prénoms ou des analyses plus ou moins fines sur les prénoms de tel ou tel ville ou village. Elle n'a malheureusement pas eu recours aux manuscrits, c'est-à-dire aux registres de naissance de l'époque, sources de première main. La nomenclature est donc la compilation de celles qu'ont constituées une centaine d'auteurs, chacun selon ses propres critères, l'un retenant un prénom comme révolutionnaire, l'autre l'excluant, le plus souvent d'ailleurs pour un motif identique. L'uniformité des relevés et des choix qui y ont présidé fait défaut, ce qui grève fortement la compilation finale. L'analyse de chaque auteur étant le plus souvent très personnelle, N.Hörsch a eu quelques difficultés à débrouiller la réalité. acceptant pour révolutionnaires tous les prénoms que ces auteurs avaient eux-mêmes considérés comme tels. Il y a là, nous semble-t-il, une erreur méthodologique dommageable : il convenait de constituer un corpus de données autonome en relevant tous les prénoms sans exclusion d'aucun- portés sur les registres de naissance de l'époque. Surtout 1793, an IV, de les comparer entre eux puis de les confronter à ceux mentionnés dans la littérature secondaire, en procédant de même pour l'analyse des données. Une telle méthode aurait présenté l'avantage de partir d'une base exhaustive et saine, dénuée de tout préjugé d'analyse. Une analyse ne peut être rigoureusement scientifique qu'à la première condition de reposer sur un corpus de données scientifiquement établi.

12 La première partie [1-60] retrace tout ce qui fait l'ambiance de la période révolutionnaire, stricto sensu. Les rappels et nombreux détails historiques dont elle fourmille forment une excellente démonstration de l'auteur, permettant notamment de mieux appréhender le climat social, politique et idéologique de l'époque. C'est la partie la plus consistante en teneur scientifique, brillamment menée. Il eût été, néanmoins, légitime de s'interroger sur la période antérieure: la Révolution n'est pas un phénomène spontané et les nombreux traits qui la caractérisent, de même que ceux qui ont contribué à l'éclosion des prénoms dits révolutionnaires, ne sont pas le fait d'une génération spontanée: ils étaient en germe depuis longtemps et les événement politiques ont permis l'ouverture des institutions à la réalité, le Droit étant, comme toujours, à la traîne de la société. Seule la diachronie peut permettre d'analyser et les événements et leurs fruits - les prénoms en l'occurrence.

La seconde partie [62-343] constitue le corps de l'ouvrage: une recherche sur les prénoms organisée selon un classement de type classique largement débiteur de la littérature secondaire. L'auteur y étudie notamment les noms de guerre de l'Ancien Régime pour les utiliser dans leur rapport avec les noms révolutionnaires : que ne l'a-telle fait pour les noms de baptême du XviII ${ }^{e}$ siècle, qui lui eussent permis d'éviter de qualifier de révolutionnaires des noms qui n'en ont strictement aucun trait? L'analyse d'un phénomène purement temporaire nécessite l'analyse des phénomènes qui l'ont précédé. Tous les rédacteurs de dictionnaires étymologiques de noms de famille et autres amateurs d'anthroponymie puiseront là de quoi méditer et réviser bon nombre 
de leurs étymologies et de leurs explications : nombre de NF sont en effet datables de cette période. Et le fait que seulement un faible pourcentage d'enfants trouvés parvenaient à l'âge adulte a empêché que le nombre en fût bien supérieur. Mais ce n'est pas parce que Pioche, nom extrait du calendrier républicain, a été conféré comme nom à des enfants trouvés qu'il est toujours d'origine révolutionnaire certaines familles le portaient auparavant.

La troisième partie [344-382] contient d'intéressants développements aréologiques, statistiques, linguistiques et historiques sur les noms, leurs bailleurs, ainsi que sur les noms des enfants trouvés. À propos des statistiques, il est regrettable que l'auteur en construise de nouvelles en compilant celles qu'elle a extraites de sources qui ne sont que de seconde main: les auteurs qu'elle a sollicités ont en effet bâti leurs propres statistiques à partir de noms subjectivement retenus comme révolutionnaires et d'après des critères infiniment variables (mêlant en outre enfants mâles et femelles, ou enfants légitimes, naturels et trouvés... ), ce qui les rend virtuellement erronées et, partant, difficilement réutilisables.

15 La quatrième partie [383-400] porte sur la liberté de nommer : les parents sont souvent contraints par les officiers publics, la sage-femme ou le chirurgien accoucheur, les témoins, la Société populaire, voire entraînés, sinon soumis, par l'ambiance régnante, certains allant même jusqu'à croire que «l'usage des nouveaux saints » proposés à la dévotion populaire par le calendrier républicain était obligatoire dans le cadre de la nomination des enfants nouveau-nés, sinon des adultes. Les nombreux textes de l'époque que l'auteur reproduit sont très parlants à cet égard, et révélateurs de cet état où la liberté, comme toujours, est l'apanage des seuls puissants du moment, rappelant que le citoyen de base n'arrivait pas à faire la différence entre le « Ferme ta gueule !» de la Monarchie et le «Cause toujours, mais surveille tes propos !» du régime qui lui a succédé. L'examen des registres montre parfaitement, à travers certains actes et autres rectifications d'actes, comme était particulièrement difficile, à cette époque, l'apprentissage de la liberté que le cadre de la loi allait vite ne pas tarder à délimiter c'est-à-dire limiter.

16 La cinquième partie [401-418] porte sur les suites de cette révolution onomastique sous les périodes postérieures. Mais peut-on vraiment parier de révolution? Pour l'auteur, oui Cependant, l'étude des noms de baptême antérieurs à la Révolution montre de façon patente et indéniable que l'évolution était largement entamée depuis de nombreuses décennies, sous la poussée notamment des littérateurs, des philosophes, de la bourgeoisie et de l'aristocratie éclairées ou protestantes, et que les événements politiques n'ont fait qu'amplifier le phénomène. Le laïque calquant en tous points le religieux, modèle omniprésent, permanent et rémanent.

17 La dernière partie [419-593] est la liste alphabétique des prénoms extraits de la littérature secondaire avec, si possible, mention du sexe, de la date, du département, du lieu, de la profession du père, de la légitimité de l'enfant, d'une éventuelle rectification de l'acte de naissance, de la fréquence du prénom et de la source. L'idée de réaliser ce répertoire est excellente car il est appelé à rendre de grands services. Mais le fait d'en avoir ordonné les prénoms par ordre alphabétique du premier prénom en cas de prénom multiple (par exemple Yves Marie Pascal Hêtre est classé sous $Y$ ) et non du prénom révolutionnaire (il devrait figurer sous $H$ ) en rend la lecture malaisée : ainsi, pour retrouver cet exemple, il faut chercher Hêtre dans l'index des noms étudiés [612-624], se reporter à la page indiquée [123] où l'on trouve alors la mention. Yves 
Marie Pascal Hêtre; pour savoir de quelle source est tiré l'exemple, il convient alors de retourner à la liste des noms [1421-593] où l'on trouve enfin la référence complète à la lettre $Y$. Si la Révolution est un long combat, la recherche d'un prénom révolutionnaire peut parfois s'apparenter à un parcours du combattant.

18 Concernant les noms eux-mêmes qui sont à la base du système bâti par l'auteur, nous ne pouvons dresser une liste de tous ceux qui appellent une remarque nécessaire sur l'interprétation qui en est faite. Nous nous bornerons simplement à remarquer qu'il nous parait difficile de pouvoir admettre comme révolutionnaires de nombreux noms comme : Agricole, Aimable, Alexandre, Ami, Angélique, Anibal, Apollonie, etc. qui ne le sont pas plus que Pierre, Paul, Jacques ou Jean. La définition était donc vraiment nécessaire pour servir de guide à l'étude.

19 L'auteur a fourni là un travail considérable et digne d'intérêt, singulièrement historique, richement documenté et analysé. Elle nous offre la première étude d'envergure sur les prénoms dits révolutionnaires, très fine dans les détails et doit en être grandement et sincèrement remerciée : son travail fournira matière à réflexion à ses lecteurs que nous souhaitons nombreux. Ils en tireront le plus grand profit.

\section{NOTES}

1.Reprise d'un compte rendu publié dans la Revue de linguistique romane, LX, 1996, pp. 593-596. 